\title{
Article \\ Companion to the Ostrowski-Grüss-Type Inequality of the Chebyshev Functional with an Application
}

\author{
Sanja Kovač ${ }^{1, *(1)}$ and Ana Vukelić ${ }^{2}$
}

check for

updates

Citation: Kovač, S.; Vukelić, A Companion to the

Ostrowski-Grüss-Type Inequality of the Chebyshev Functional with an Application. Mathematics 2022, 10, 735. https://doi.org/10.3390/ math10050735

Academic Editor: Juan Benigno Seoane-Sepúlveda

Received: 31 December 2021 Accepted: 24 February 2022

Published: 25 February 2022

Publisher's Note: MDPI stays neutral with regard to jurisdictional claims in published maps and institutional affiliations.

Copyright: (C) 2022 by the authors. Licensee MDPI, Basel, Switzerland. This article is an open access article distributed under the terms and conditions of the Creative Commons Attribution (CC BY) license (https:// creativecommons.org/licenses/by/ $4.0 /)$.
1 Faculty of Geotechnical Engineering, University of Zagreb, 42000 Varaždin, Croatia

2 Faculty of Food and Biotechnology, University of Zagreb, 10000 Zagreb, Croatia; avukelic@pbf.unizg.hr

* Correspondence: sanja.kovac@gfv.unizg.hr

\begin{abstract}
Recently, there have been many proven results of the Ostrowski-Grüss-type inequality regarding the error bounds for the Chebyshev functional when the functions or their derivatives belong to $L_{p}$ spaces. In the existing literature, the main assumption in the weight-type results is that the derivative of the function is bounded by two constant functions. The aim of our paper is to extend those results in a way that the derivative of the function is bounded by two functions in $L_{p}$ spaces. Furthermore, we give some new error estimations of the Chebyshev functional and applications to the one-point weight integral formulas.
\end{abstract}

Keywords: Chebyshev functional; Grüss inequality; Ostrowski inequality; weight integral formula

MSC: 26D15; 65D30; 65D32

\section{Introduction}

Throughout this paper, $L_{p}[a, b], 1 \leq p<\infty$ stands for the space of the functions $f:[a, b] \rightarrow \mathbb{R}$, which are $p$-integrable, which means that they are equipped with the p-norm:

$$
\|f\|_{p}=\left[\int_{a}^{b}|f(t)|^{p} d t\right]^{\frac{1}{p}}
$$

which is finite. $L_{\infty}[a, b]$ stands for the space of the functions $f:[a, b] \rightarrow \mathbb{R}$, which are essentially bounded, i.e., the $\infty$-norm defined by:

$$
\|f\|_{\infty}=\operatorname{esssup}_{t \in[a, b]}|f(t)|
$$

is finite.

Let $f, g:[a, b] \rightarrow \mathbb{R}$ be the functions such that $f, g, f \cdot g \in L_{1}[a, b]$. The Chebyshev functional $T(f, g)$ is defined by:

$$
T(f, g):=\frac{1}{b-a} \int_{a}^{b} f(x) g(x) d x-\frac{1}{b-a} \int_{a}^{b} f(x) d x \cdot \frac{1}{b-a} \int_{a}^{b} g(x) d x .
$$

Let us recall the Grüss inequality ([1]):

Theorem 1. Let $f, g:[a, b] \rightarrow \mathbb{R}$ be bounded integrable functions and $\varphi, \Phi, \gamma, \Gamma \in \mathbb{R}$ constants such that:

$$
\varphi \leq f(x) \leq \Phi \quad \text { and } \quad \gamma \leq g(x) \leq \Gamma, \quad \forall x \in[a, b]
$$

Then, the following inequality holds:

$$
|T(f, g)| \leq \frac{1}{4}(\Phi-\varphi)(\Gamma-\gamma)
$$


where the constant $\frac{1}{4}$ is sharp.

The following theorem recalls the well-known Ostrowski inequality, which was established in 1938 [2]:

Theorem 2. Let I be an interval in $\mathbb{R}, I^{0}$ its interior, and $a, b \in I^{o}$ with $a<b$. If $f: I \rightarrow \mathbb{R}$ is a differentiable function in $I^{o}$ satisfying $\left|f^{\prime}(t)\right| \leq M$, for all $t \in[a, b]$ and some $M>0$, then:

$$
\left|f(x)-\frac{1}{b-a} \int_{a}^{b} f(t) d t\right| \leq\left[\frac{1}{4}+\frac{\left(x-\frac{a+b}{2}\right)^{2}}{(b-a)^{2}}\right](b-a) M,
$$

for all $x \in[a, b]$.

Many researchers have established some new results by using the Grüss and Ostrowski inequalities and given applications to the numerical quadrature rules [3-8].

Dragomir and Wang [3] proved the Ostrowski-Grüss-type inequality in the following form: If $f:[a, b] \rightarrow \mathbb{R}$ is a differentiable function with a bounded derivative and:

$$
\alpha_{0} \leq f^{\prime}(t) \leq \beta_{0}, \quad t \in[a, b],
$$

then for $x \in[a, b]$, we have:

$$
\left|f(x)-\frac{1}{b-a} \int_{a}^{b} f(t) d t-\frac{f(b)-f(a)}{b-a}\left(x-\frac{a+b}{2}\right)\right| \leq \frac{1}{4}(b-a)\left(\beta_{0}-\alpha_{0}\right) .
$$

Matić, Pečarić, and Ujević [4] proved the following Ostrowski-Grüss-type inequality: Let $f: I \rightarrow \mathbb{R}$ be $n$-times differentiable in the interior $I^{0}$ of $I$, and let $a, b \in I^{0}$ with $a<b$. If $f^{(n)}$ is integrable on $[a, b]$ and:

$$
\gamma \leq f^{(n)}(t) \leq \Gamma, \quad t \in[a, b]
$$

then for all $x \in[a, b]$,

$$
\left|R_{n}(x)\right| \leq \frac{\Gamma-\gamma}{2(n !)}\left[\frac{(b-x)^{n+1}+(-1)^{n}(x-a)^{n+1}}{(b-a)(2 n+1)}-\left(\frac{(x-a)^{n+1}-(x-b)^{n+1}}{(b-a)(n+1)}\right)^{2}\right] .
$$

The remainder $R_{n}(x)$ is defined by:

$$
\begin{aligned}
& R_{n}(x)=f(x)+\frac{1}{b-a} \sum_{k=1}^{n-1} \frac{(b-x)^{k-1}+(-1)^{k}(x-a)^{k+1}}{(k+1) !} f^{(k)}(x) \\
& +\frac{(b-x)^{n+1}+(-1)^{n}(x-a)^{n+1}}{(n+1) !(b-a)^{2}}\left[f^{(n-1)}(b)-f^{(n-1)}(a)\right]-\frac{1}{b-a} \int_{a}^{b} f(t) d t
\end{aligned}
$$

For the special case $n=1$, we have:

$$
\left|f(x)-\frac{1}{b-a} \int_{a}^{b} f(t) d t-\frac{f(b)-f(a)}{b-a}\left(x-\frac{a+b}{2}\right)\right| \leq \frac{1}{4 \sqrt{3}}(b-a)(\Gamma-\gamma)
$$

for all $x \in \mathbb{R}$.

Cheng [9] proved the following Ostrowski-Grüss-type inequality: Let $f:[a, b] \rightarrow \mathbb{R}$ be a differentiable mapping in $(a, b)$ such that $\gamma_{1} \leq f^{\prime}(x) \leq \Gamma_{1}$, then for all $x \in[a, b]$, we have:

$$
\left|f(x)-\frac{1}{b-a} \int_{a}^{b} f(t) d t-\frac{f(b)-f(a)}{b-a}\left(x-\frac{a+b}{2}\right)\right| \leq \frac{1}{8}(b-a)\left(\Gamma_{1}-\gamma_{1}\right) .
$$

Niezgoda [10] established the following result: 
Theorem 3. Let $f: I \subset \mathbb{R} \rightarrow \mathbb{R}$ be a function differentiable in the interior $I^{o}$ of $I$, and let $[a, b] \subset I^{o}$. Suppose that $f^{\prime}, \alpha, \beta \in L_{p}[a, b](1 \leq p \leq \infty)$ are functions such that:

(a) $\alpha+\beta$ is a constant function;

(b) $\quad \alpha(t) \leq f^{\prime}(t) \leq \beta(t)$, for all $t \in[a, b]$.

Then, for $x \in[a, b]$, we have the inequality:

$$
\begin{aligned}
& \left|f(x)-\frac{1}{b-a} \int_{a}^{b} f(t) d t-\left(x-\frac{a+b}{2}\right) \frac{f(b)-f(a)}{b-a}\right| \\
& \leq \begin{cases}\frac{1}{4}\|\beta-\alpha\|_{p} \frac{(b-a)^{1 / q}}{(q+1)^{1 / q}}, & \text { if } 1 \leq q<\infty \\
\frac{1}{4}\|\beta-\alpha\|_{1}, & \text { if } q=\infty,\end{cases}
\end{aligned}
$$

where $\frac{1}{p}+\frac{1}{p}=1$.

Kovač and Pečarić [11] gave the general weight m-point integral formula:

Theorem 4. Let $g:[a, b] \rightarrow \mathbb{R}$ be such that $g^{(n-1)}$ is a continuous function of bounded variation on $[a, b]$, for some $n \in \mathbb{N}$ and $w:[a, b] \rightarrow[0, \infty\rangle$ some integrable weight function. Then, the following identity holds:

$$
\begin{aligned}
\int_{a}^{b} w(t) g(t) d t & =\sum_{j=1}^{n}(-1)^{j-1}\left[w_{m j}(b) g^{(j-1)}(b)\right. \\
& \left.+\sum_{k=1}^{m-1}\left[w_{k j}\left(x_{k}\right)-w_{k+1, j}\left(x_{k}\right)\right] g^{(j-1)}\left(x_{k}\right)-w_{1 j}(a) g^{(j-1)}(a)\right] \\
& +(-1)^{n} \int_{a}^{b} W_{n, w}(t, \sigma) d g^{(n-1)}(t) .
\end{aligned}
$$

Here, $\left\{w_{k j}\right\}_{j=1, \ldots, n}$ are $w$-harmonic sequences of functions $\left(\left(w_{k j}\right)^{\prime}(t)=w_{k, j-1}(t)\right.$ for $\left.t \in\left[x_{k-1}, x_{k}\right], k=1, \ldots, m\right)$ :

$$
W_{n, w}(t, \sigma)= \begin{cases}w_{1 n}(t) & \text { for } t \in\left[a, x_{1}\right], \\ w_{2 n}(t) & \text { for } t \in\left(x_{1}, x_{2}\right], \\ \vdots & \\ w_{m n}(t) & \text { for } t \in\left(x_{m-1}, b\right] .\end{cases}
$$

In the same paper, the authors established the weighted one-point integral formula as a special case:

$$
\int_{a}^{b} w(t) f(t) d t=\sum_{j=1}^{n} A_{w, j}(x) f^{(j-1)}(x)+(-1)^{n} \int_{a}^{b} W_{n, w}(t, x) f^{(n)}(t) d t
$$

where $f:[a, b] \rightarrow \mathbb{R}$ is such that $f^{(n-1)}$ is an absolutely continuous function, $w:[a, b] \rightarrow$ $[0, \infty)$ is a weight function, $x \in[a, b]$ :

$$
A_{w, j}(x)=\frac{(-1)^{j-1}}{(j-1) !} \int_{a}^{b}(x-s)^{j-1} w(s) d s, \quad \text { for } j=1, \ldots, n
$$

and:

$$
W_{n, w}(t, x)= \begin{cases}w_{1 n}(t)=\frac{1}{(n-1) !} \int_{a}^{t}(t-s)^{n-1} w(s) d s & \text { for } t \in[a, x], \\ w_{2 n}(t)=\frac{1}{(n-1) !} \int_{b}^{t}(t-s)^{n-1} w(s) d s & \text { for } t \in(x, b] .\end{cases}
$$


The aim of our paper was to give the generalization of Niezgoda's result by using the general weight $m$-point integral formula. Further, we shall give some applications to the one-point integral formula.

\section{Main Result}

First, let us denote:

$$
\begin{aligned}
\operatorname{Tg}\left(x_{0}, x_{1}, \ldots, x_{m}\right) & :=\frac{1}{b-a}\left[\int_{a}^{b} w(t) g(t) d t-\sum_{j=1}^{n}(-1)^{j-1}\left[w_{m j}(b) g^{(j-1)}(b)\right.\right. \\
& \left.\left.+\sum_{k=1}^{m-1}\left[w_{k j}\left(x_{k}\right)-w_{k+1, j}\left(x_{k}\right)\right] g^{(j-1)}\left(x_{k}\right)-w_{1 j}(a) g^{(j-1)}(a)\right]\right]
\end{aligned}
$$

and:

$$
\eta=\frac{(-1)^{n}}{b-a} \int_{a}^{b} W_{n, w}(t, \sigma) d t .
$$

Let us consider the Chebyshev functional for functions $(-1)^{n} W_{n, w}(\cdot, \sigma)$ and $g^{(n)}$ :

$$
T\left((-1)^{n} W_{n, w}(\cdot, \sigma), g^{(n)}\right)=T g\left(x_{0}, x_{1}, \ldots, x_{m}\right)-\frac{(-1)^{n}}{b-a} \int_{a}^{b} W_{n, w}(t, \sigma) d t \cdot \frac{1}{b-a} \int_{a}^{b} g^{(n)}(t) d t .
$$

This section's objective is to determine the upper bound for (15). We use the following lemma (see [10]) to prove our main theorem:

Lemma 1. Let $\alpha, \beta \in L_{p}[a, b](1 \leq p \leq \infty)$ be a function such that:

$$
\alpha(t) \leq f(t) \leq \beta(t), \quad \forall t \in[a, b] .
$$

Then, we have the inequality:

$$
\left\|f-\frac{\alpha+\beta}{2}\right\|_{p} \leq \frac{1}{2}\|\beta-\alpha\|_{p}
$$

Now, we are ready to introduce our main result:

Theorem 5. Let $g: I \subset \mathbb{R} \rightarrow \mathbb{R}$ be a function such that $g^{(n-1)}$ is differentiable in the interior $I^{o}$ of $I$, and let $[a, b] \subset I^{o}$. Suppose that $g^{(n)}, \alpha, \beta \in L_{p}[a, b](1 \leq p \leq \infty)$ are functions such that:

(a) $\alpha+\beta$ is a constant function;

(b) $\quad \alpha(t) \leq g^{(n)}(t) \leq \beta(t)$, for all $t \in[a, b]$.

Then, the following inequality holds:

$$
\begin{aligned}
& \mid \frac{1}{b-a} \int_{a}^{b} w(t) g(t) d t-\frac{1}{b-a} \sum_{j=1}^{n}(-1)^{j-1}\left[w_{m j}(b) g^{(j-1)}(b)\right. \\
+ & \left.\sum_{k=1}^{m-1}\left[w_{k j}\left(x_{k}\right)-w_{k+1, j}\left(x_{k}\right)\right] g^{(j-1)}\left(x_{k}\right)-w_{1 j}(a) g^{(j-1)}(a)\right] \\
- & \frac{g^{(n-1)}(b)-g^{(n-1)}(a)}{b-a} \cdot \eta \mid \\
\leq & \frac{1}{2(b-a)}\|\beta-\alpha\|_{p} \cdot\left\|(-1)^{n} W_{n, w}(\cdot, \sigma)-\eta\right\|_{q}
\end{aligned}
$$

where $\frac{1}{p}+\frac{1}{p}=1$.

Proof. From the identity (15), we have: 


$$
\begin{aligned}
& T\left((-1)^{n} W_{n, w}(\cdot, \sigma), g^{(n)}\right)= \\
= & \frac{1}{b-a} \int_{a}^{b} w(t) g(t) d t-\frac{1}{b-a} \sum_{j=1}^{n}(-1)^{j-1}\left[w_{m j}(b) g^{(j-1)}(b)\right. \\
+ & \left.\sum_{k=1}^{m-1}\left[w_{k j}\left(x_{k}\right)-w_{k+1, j}\left(x_{k}\right)\right] g^{(j-1)}\left(x_{k}\right)-w_{1 j}(a) g^{(j-1)}(a)\right] \\
- & \frac{g^{(n-1)}(b)-g^{(n-1)}(a)}{b-a} \cdot \eta .
\end{aligned}
$$

Let us apply the Sonin identity (see [2], p. 246). For $c \in \mathbb{R}$,

$$
T\left((-1)^{n} W_{n, w}(\cdot, \sigma), g^{(n)}\right)=\frac{1}{b-a} \int_{a}^{b}\left(g^{(n)}(t)-c\right) \cdot\left((-1)^{n} W_{n, w}(t, \sigma)(t)-\eta\right) d t .
$$

Now, we apply the Hölder inequality to the identity (16) to obtain:

$$
\left|T\left((-1)^{n} W_{n, w}(\cdot, \sigma), g^{(n)}\right)\right| \leq \frac{1}{b-a}\left\|g^{(n)}-c\right\|_{p} \cdot\left\|(-1)^{n} W_{n, w}(\cdot, \sigma)-\eta\right\|_{q} .
$$

Since $\alpha+\beta$ is a constant function, we can substitute $c=\frac{\alpha+\beta}{2}$, and by Lemma 1 , we obtain:

$$
\begin{aligned}
\left|T\left((-1)^{n} W_{n, w}(\cdot, \sigma), g^{(n)}\right)\right| & \leq \frac{1}{b-a}\left\|g^{(n)}-\frac{\alpha+\beta}{2}\right\|_{p} \cdot\left\|W_{n, w}(\cdot, \sigma)-\eta\right\|_{q} \\
& \leq \frac{1}{2(b-a)}\|\beta-\alpha \mid\|_{p} \cdot\left\|(-1)^{n} W_{n, w}(\cdot, \sigma)-\eta\right\|_{q}
\end{aligned}
$$

which completes the proof.

Now, we shall consider the special case where $\alpha$ and $\beta$ are constant functions.

Corollary 1. Let $g: I \subset \mathbb{R} \rightarrow \mathbb{R}$ be a function such that $g^{(n-1)}$ is differentiable in the interior $I^{o}$ of $I$, and let $[a, b] \subset I^{o}$. Suppose that $\alpha_{0}, \beta_{0} \in \mathbb{R}$ such that $\alpha_{0} \leq g^{(n)}(t) \leq \beta_{0}$ for all $t \in[a, b]$. Then, the following inequality holds:

$$
\begin{aligned}
& \mid \frac{1}{b-a} \int_{a}^{b} w(t) g(t) d t-\frac{1}{b-a} \sum_{j=1}^{n}(-1)^{j-1}\left[w_{m j}(b) g^{(j-1)}(b)\right. \\
& \left.+\sum_{k=1}^{m-1}\left[w_{k j}\left(x_{k}\right)-w_{k+1, j}\left(x_{k}\right)\right] g^{(j-1)}\left(x_{k}\right)-w_{1 j}(a) g^{(j-1)}(a)\right] \\
& -\frac{g^{(n-1)}(b)-g^{(n-1)}(a)}{b-a} \cdot \eta \mid \\
& \leq \begin{cases}\frac{\beta_{0}-\alpha_{0}}{2(b-a)^{1 / q}} \cdot\left\|(-1)^{n} W_{n, w}(\cdot, \sigma)-\eta\right\|_{q}, & \text { for } 1 \leq q<\infty, \\
\frac{\beta_{0}-\alpha_{0}}{2} \cdot\left\|(-1)^{n} W_{n, w}(\cdot, \sigma)-\eta\right\|_{q}, & \text { for } q=\infty\end{cases}
\end{aligned}
$$

where $\frac{1}{p}+\frac{1}{p}=1$.

Proof. Let $1 \leq p, q \leq \infty$ be conjugate exponents, i.e., $\frac{1}{p}+\frac{1}{q}=1$. It is obvious that $f^{\prime} \in L_{\infty}[a, b] \subset L_{p}[a, b]$. Substituting functions $\alpha(t)$ and $\beta(t)$ with constant functions $\alpha_{0}$ and $\beta_{0}$ in Theorem 5 , it is easy to check that Conditions (a) and (b) from Theorem 5 are valid. Consequently, Inequality (16) holds. For $1 \leq p<\infty$, we compute:

$$
\|\beta-\alpha\|_{p}=\left(\beta_{0}-\alpha_{0}\right) \cdot(b-a)^{1 / p}
$$


and for $p=\infty$, we have:

$$
\|\beta-\alpha\|_{\infty}=\beta_{0}-\alpha_{0} .
$$

Therefore, for $1 \leq q<\infty$, the right-hand side of (16) equals:

$$
\frac{\left(\beta_{0}-\alpha_{0}\right)}{2(b-a)^{1 / q}} \cdot\left\|(-1)^{n} W_{n, w}(\cdot, \sigma)-\eta\right\|_{q}
$$

while for $q=\infty$, it equals:

$$
\frac{\beta_{0}-\alpha_{0}}{2} \cdot\left\|(-1)^{n} W_{n, w}(\cdot, \sigma)-\eta\right\|_{q} .
$$

Remark 1. The hypothesis that the $n$-th derivative of the function is bounded by two constants $\alpha_{0}$ and $\beta_{0}$ is more general than the hypothesis where the $n$-th derivative is bounded by the functions $\alpha(t)$ and $\beta(t)$. Therefore, if we assume that the conditions from the Theorem 5 and Corollary 1 are satisfied with:

$$
\alpha_{0} \leq \beta(t) \leq g^{(n)}(t) \leq \beta(t) \leq \beta_{0},
$$

the constant $\frac{1}{2(b-a)}\|\beta-\alpha\|_{p}$ is smaller than the constant $\frac{\beta_{0}-\alpha_{0}}{2(b-a)^{1 / q}}$, for $1 \leq q<\infty$, and $\frac{\beta_{0}-\alpha_{0}}{2}$, for $q=\infty$. Namely, for $1 \leq p<\infty$, we have:

$$
\frac{1}{2(b-a)}\|\beta-\alpha\|_{p}=\frac{1}{b-a}\left[\int_{a}^{b}(\beta(t)-\alpha(t))^{p} d t\right]^{1 / p} \leq \frac{\beta_{0}-\alpha_{0}}{2(b-a)^{1 / q}},
$$

while for $p=\infty$, we have:

$$
\frac{\|\beta-\alpha\|_{\infty}}{2(b-a)} \leq \frac{\beta_{0}-\alpha_{0}}{2(b-a)} .
$$

\section{Application to the One-Point Integral Formula}

In this section, we shall apply the result from Section 2 to the case $m=2$, i.e., when we have the one-point integral formula. First, we consider the general weight case and higher-order derivative.

Corollary 2. Let $g: I \subset \mathbb{R} \rightarrow \mathbb{R}$ be a function such that $g^{(n-1)}$ is differentiable in the interior $I^{o}$ of $I$, and let $[a, b] \subset I^{o}$. Suppose that $g^{(n)}, \alpha, \beta \in L_{p}[a, b](1 \leq p \leq \infty)$ are functions such that:

(a) $\alpha+\beta$ is a constant function;

(b) $\quad \alpha(t) \leq g^{(n)}(t) \leq \beta(t)$, for all $t \in[a, b]$.

Then, the following inequality holds:

$$
\begin{aligned}
& \quad\left|\frac{1}{b-a} \int_{a}^{b} g(t) w(t) d t-\frac{1}{b-a} \sum_{j=1}^{n} A_{j}(x) g^{(j-1)}(x)-\frac{g^{(n-1)}(b)-g^{(n-1)}(a)}{b-a} \cdot \eta_{1}\right| \\
& \leq \frac{1}{2(b-a)}\|\beta-\alpha\|_{p} \cdot\left\|(-1)^{n} W_{n, w}(\cdot x)-\eta_{1}\right\|_{q},
\end{aligned}
$$

where $\frac{1}{p}+\frac{1}{p}=1$ and $\eta_{1}=\frac{(-1)^{n}}{b-a} \int_{a}^{b} W_{n, w}(t, x) d t$.

Proof. We apply Theorem 5 for $m=2, x_{0}=a, x_{1}=x$, and $x_{2}=b$ to obtain the inequality. 
Now, we shall consider the case of uniform weight function $\left(w(t)=\frac{1}{b-a}\right)$. For this case, we have:

$$
\begin{gathered}
W_{n}(t, x)= \begin{cases}w_{1 n}(t)=\frac{(t-a)^{n}}{n !} & \text { for } t \in[a, x], \\
w_{2 n}(t)=\frac{(t-b)^{n}}{n !} & \text { for } t \in(x, b],\end{cases} \\
A_{j}(x)=\frac{(-1)^{j-1}}{j !(b-a)}\left[(x-b)^{j}-(x-a)^{j}\right]
\end{gathered}
$$

and:

$$
\eta_{1}=\frac{(-1)^{n}\left[(x-a)^{n+1}-(x-b)^{n+1}\right]}{(b-a) \cdot(n+1) !} .
$$

Corollary 3. Let $g: I \subset \mathbb{R} \rightarrow \mathbb{R}$ be a function such that $g^{(n-1)}$ is differentiable in the interior $I^{o}$ of $I$, and let $[a, b] \subset I^{o}$. Suppose that $g^{(n)}, \alpha, \beta \in L_{p}[a, b](1 \leq p \leq \infty)$ are functions such that:

(a) $\alpha+\beta$ is a constant function;

(b) $\quad \alpha(t) \leq g^{(n)}(t) \leq \beta(t)$, for all $t \in[a, b]$.

Then, the following inequality holds:

$$
\begin{aligned}
& \quad \mid \frac{1}{b-a} \int_{a}^{b} g(t) d t-\frac{1}{b-a} \sum_{j=1}^{n} \frac{(-1)^{j}}{j !}\left[(x-b)^{j}-(x-a)^{j}\right] g^{(j-1)}(x) \\
& \quad-\frac{g^{(n-1)}(b)-g^{(n-1)}(a)}{b-a} \cdot \eta_{1} \mid \\
& \leq \frac{1}{2(b-a)}\|\beta-\alpha\|_{p} \cdot\left\|(-1)^{n} W_{n}(\cdot, x)-\eta_{1}\right\|_{q},
\end{aligned}
$$

where $\frac{1}{p}+\frac{1}{p}=1$.

Remark 2. If we put $m=2, x_{0}=a, x_{1}=x, x_{2}=b$, and $n=1$ in Corollary 3 , then we have the assumptions of Theorem 3. In this case:

$$
\eta_{1}=\frac{a+b}{2}-x
$$

and Inequality (22) states:

$$
\begin{aligned}
& \left|g(x)-\frac{1}{b-a} \int_{a}^{b} g(t) d t-\left(x-\frac{a+b}{2}\right) \frac{g(b)-g(a)}{b-a}\right| \\
& \leq \frac{1}{2(b-a)}\|\beta-\alpha\|_{p} \cdot\left\|W_{1}(\cdot, x)+\eta_{1}\right\|_{q},
\end{aligned}
$$

Without loss of generality, we can assume that $a \leq x \leq \frac{a+b}{2}$. Therefore, for $t \in[a, x)$, we have $t-a+\frac{a+b}{2}-x \geq 0$, for $t \in\left[x, x+\frac{b-a}{2}\right)$, we have $t-b+\frac{a+b}{2}-x \leq 0$, and for $t \in\left[x+\frac{b-a}{2}, b\right]$, we have $t-b+\frac{a+b}{2}-x \geq 0$. Now, for $1 \leq q<\infty$, we have: 


$$
\begin{aligned}
& \left\|W_{1}(\cdot, x)+\eta_{1}\right\|_{q}=\left\|W_{1}(\cdot, x)+\frac{a+b}{2}-x\right\|_{q}= \\
& =\left[\int_{a}^{x}\left|t-a+\frac{a+b}{2}-x\right|^{q} d t+\int_{x}^{b}\left|t-b+\frac{a+b}{2}-x\right|^{q} d t\right]^{1 / q}= \\
& =\left[\int_{a}^{x}\left(t+\frac{b-a}{2}-x\right)^{q} d t+\int_{x}^{x+\frac{b-a}{2}}\left(-t+\frac{b-a}{2}+x\right)^{q} d t\right. \\
& \left.+\int_{x+\frac{b-a}{2}}^{b}\left(t-\frac{b-a}{2}-x\right)^{q} d t\right]^{1 / q}= \\
& =\frac{(b-a)^{1+1 / q}}{2 \cdot(q+1)^{1 / q}}
\end{aligned}
$$

For $q=\infty$, it is easy to check that:

$$
\left\|W_{1}(\cdot, x)+\eta_{1}\right\|_{\infty}=\frac{b-a}{2}
$$

Therefore, it is shown that our main result is the improvement of Niezgoda's result mentioned in the Introduction.

Corollary 4. Let $g:[a, b] \rightarrow \mathbb{R}$ be a differentiable function with a bounded derivative. Suppose that $g^{\prime}, \alpha, \beta \in L_{1}[a, b]$ are functions such that:

(a) $\alpha+\beta$ is a constant function:

(b) $\quad \alpha(t) \leq g^{\prime}(t) \leq \beta(t)$, for all $t \in[a, b]$.

Then, for $x \in[a, b]$ we have:

$$
\left|g(x)-\frac{1}{b-a} \int_{a}^{b} g(t) d t-\left(x-\frac{b-a}{2}\right) \frac{g(b)-g(a)}{b-a}\right| \leq \frac{1}{4}\|\beta-\alpha\|_{1} .
$$

Proof. The assertion follows if we apply $n=1, q=\infty$, and $p=1$ in Corollary 3 .

Remark 3. If we put constant functions $\alpha_{0}$ and $\beta_{0}$, then we will obtain:

$$
\left\|\beta_{0}-\alpha_{0}\right\|_{1}=\left(\beta_{0}-\alpha_{0}\right) \cdot(b-a) .
$$

This is the Ostrowski-Grüss-type inequality obtained by Dragomir and Wang (3). Therefore, Corollary 4 is the improvement of Dragomir-Wang's result.

Corollary 5. Let $g: I \rightarrow \mathbb{R}$ be a function differentiable on the interior $I^{o}$ of $I$, and let $a, b \in I^{o}$ with $a<b$. Suppose that $g^{\prime}, \alpha, \beta \in L_{2}[a, b]$ are functions such that:

(a) $\alpha+\beta$ is a constant function:

(b) $\alpha(t) \leq g^{\prime}(t) \leq \beta(t)$, for all $t \in[a, b]$.

Then, the following inequality holds:

$$
\left|g(x)-\frac{1}{b-a} \int_{a}^{b} g(t) d t-\left(x-\frac{b-a}{2}\right) \frac{g(b)-g(a)}{b-a}\right| \leq \frac{1}{4 \sqrt{3}} \sqrt{b-a}\|\beta-\alpha\|_{2} .
$$

Proof. The assertion follows if we apply $n=1, q=2$, and $p=2$ in Corollary 3 .

Remark 4. If we put constant functions $\alpha_{0}$ and $\beta_{0}$, then we will obtain:

$$
\left\|\beta_{0}-\alpha_{0}\right\|_{2}=\left(\beta_{0}-\alpha_{0}\right) \cdot \sqrt{b-a} .
$$


This is the Ostrowski-Grüss-type inequality obtained by Matić, Pečarić, and Ujević [4], so Corollary 5 is the improvement of Matić, Pečarić, and Ujević's result.

Corollary 6. Let $g:[a, b] \rightarrow \mathbb{R}$ be a differentiable function in $(a, b)$. Suppose that $\alpha, \beta:[a, b] \rightarrow$ $\mathbb{R}$ are functions such that $g^{\prime}, \alpha, \beta \in L_{\infty}[a, b]$ :

(a) $\alpha+\beta$ is a constant function;

(b) $\quad \alpha(t) \leq g^{\prime}(t) \leq \beta(t)$, for all $t \in[a, b]$.

Then, for all $x \in[a, b]$, we have:

$$
\left|g(x)-\frac{1}{b-a} \int_{a}^{b} g(t) d t-\left(x-\frac{b-a}{2}\right) \frac{g(b)-g(a)}{b-a}\right| \leq \frac{b-a}{8}\|\beta-\alpha\|_{\infty} .
$$

Proof. The assertion follows if we apply $n=1, q=1$, and $p=\infty$ in Corollary 3 .

Remark 5. If we put constant functions $\alpha_{0}$ and $\beta_{0}$, then we will obtain:

$$
\left\|\beta_{0}-\alpha_{0}\right\|_{\infty}=\left(\beta_{0}-\alpha_{0}\right) \text {. }
$$

This is the Ostrowski-Grüss-type inequality obtained by Cheng [9]. Therefore, Corollary 6 is the improvement of Cheng's result.

Especially, for $x=\frac{a+b}{2}$, we have:

$$
\eta_{1}= \begin{cases}0 & \text { for } n \text { odd } \\ \frac{(-1)^{n}(b-a)^{n}}{2^{n}(n+1) !} & \text { for } n \text { even }\end{cases}
$$

Now, we give the special case of the upper inequality related to the corrected midpoint quadrature formula (corrected in the sense that we have derivatives in endpoints):

Corollary 7. Let $g: I \subset \mathbb{R} \rightarrow \mathbb{R}$ be a function such that $g^{\prime}$ is differentiable in the interior $I^{o}$ of $I$, and let $[a, b] \subset I^{o}$. Suppose that $g^{\prime \prime}, \alpha, \beta \in L_{p}[a, b](1 \leq p \leq \infty)$ are functions such that:

(a) $\alpha+\beta$ is a constant function;

(b) $\quad \alpha(t) \leq g^{\prime \prime}(t) \leq \beta(t)$, for all $t \in[a, b]$.

Then, the following inequality holds:

$$
\begin{aligned}
& \left|\frac{1}{b-a} \int_{a}^{b} g(t) d t-g\left(\frac{a+b}{2}\right)-\frac{b-a}{24}\left[g^{\prime}(b)-g^{\prime}(a)\right]\right| \\
& \leq \begin{cases}\frac{(b-a)^{2}}{36 \sqrt{3}}\|\beta-\alpha\|_{\infty} & \text { for } q=1, p=\infty \\
\frac{(b-a)^{3}}{1440}\|\beta-\alpha\|_{2} & \text { for } q=p=2 \\
\frac{b-a}{24}\|\beta-\alpha\|_{1} & \text { for } q=\infty, p=1\end{cases}
\end{aligned}
$$

Proof. We apply Corollary 3 for $n=2$ and $x=\frac{a+b}{2}$. It is easy to calculate $A_{1}\left(\frac{a+b}{2}\right)=1$ and $A_{2}\left(\frac{a+b}{2}\right)=0$. For $q=1$, we have after some integration:

$$
\left\|W_{2}\left(t, \frac{a+b}{2}\right)-\eta_{1}\right\|_{1}=\frac{(b-a)^{2}}{18 \sqrt{3}} .
$$

For $q=2$, we compute:

$$
\left\|W_{2}\left(t, \frac{a+b}{2}\right)-\eta_{1}\right\|_{2}=\frac{(b-a)^{3}}{720} .
$$


For $q=\infty$, we have:

$$
\left\|W_{2}\left(t, \frac{a+b}{2}\right)-\eta_{1}\right\|_{\infty}=\frac{b-a}{12},
$$

and the assertion follows.

Remark 6. For $n=1$ and $x=\frac{a+b}{2}$, we obtain the error of the famous midpoint quadrature rule ([12]).

\section{Conclusions}

In this work, we established an improved version of the Ostrowski-Grüss-type inequalities obtained in $[3,4,9,10]$. The main contribution of the work was that the hypothesis that the derivative is bounded by two constant functions is replaced by the more general hypothesis where the derivative of the functions is bounded by two non-constant functions.

Author Contributions: Conceptualization, S.K; methodology, S.K.; software, S.K.; validation, S.K. and A.V.; formal analysis, S.K.; investigation, S.K.; resources, S.K.; data curation, S.K.; writingoriginal draft preparation, S.K.; writing - review and editing, S.K.; visualization, S.K.; supervision, S.K.; project administration, S.K.; funding acquisition, S.K. All authors have read and agreed to the published version of the manuscript.

Funding: This research received no external funding.

Institutional Review Board Statement: Not applicable.

Informed Consent Statement: Not applicable.

Data Availability Statement: Not applicable.

Conflicts of Interest: The authors declare no conflict of interest.

\section{References}

1. Grüss, G. Uber das maximum des absoluten betrages von $\frac{1}{b-a} \int_{a}^{b} f(x) d x \int_{a}^{b} g(x) d x$. Math. Z. 1934, 39, 215-226. [CrossRef]

2. Mitrinović, D.S.; Pečarić, J.E.; Fink, A.M. Classical and New Inequalities in Analysis; Kluwer Academic: Dordrecht, The Netherlands, 1993.

3. Dragomir, S.S.; Wang, S. An inequality of Ostrowski-Grüss' type and its applications to estimation of error bounds for some special means and for some numerical quadrature rules. Comput. Math. Appl. 1997, 39, 15-20. [CrossRef]

4. Matić, M.; Pečarić, J.; Ujević, N. Improvement and further generalization of inequalities of Ostrowski-Grüss-type. Comput. Math. Appl. 2000, 39, 161-175. [CrossRef]

5. Rehman, A.U.; Farid, G. On Chebyshev Functional and Ostrowski-Grus Type Inequalities for Two Coordinates. Int. J. Anal. Appl. 2016, 12, 180-187.

6. Hussain, S.; Qayyum, Q. A generalized Ostrowski-Grüss-type inequality for bounded differentiable mappings and its applications. J. Inequal. Appl. 2013, 2013, 1. [CrossRef]

7. Rafiq, A.; Mir, N.A.; Zafar, F. A generalized Ostrowski-Grüss-type inequality for twice differentiable mappings and applications. J. Inequalities Pure Appl. Math. 2006, 7, 124.

8. Alomari, M.W. Two-point Ostrowski and Ostrowski-Grüss-type inequalities with applications. J. Anal. $202028,623-661$. [CrossRef]

9. Cheng, X.-L. Improvement of some Ostrowski-Grüss-type inequalities. Comput. Math. Appl. 2001, 42, 109-114. [CrossRef]

10. Niezgoda, M. A new inequality of Ostrowski-Grüss-type and applications to some numerical quadrature rules. Comput. Math. Appl. 2009, 58, 589-596. [CrossRef]

11. Kovač, S.; Pečarić, J. Weighted version of general integral formula. Math. Inequal. Appl. 2010, 13, 579-599.

12. Kovač, S.; Pečarić, J.; Vukelić, A. A generalization of general two-point formula with applications in numerical integration. Nonlinear Anal. Theory Methods Appl. 2008, 68, 2445-2463. [CrossRef] 